\author{
Original article
}

\title{
Fluctuations in haemocyte density and microbial load may be used as indicators of fungal pathogenicity in larvae of Galleria mellonella
}

\author{
David Bergin, Marc Brennan, Kevin Kavanagh * \\ Department of Biology, Medical Mycology Unit, National Institute for Cellular Biotechnology, National University of Ireland Maynooth, \\ Co. Kildare, Ireland
}

Received 7 July 2003; accepted 17 September 2003

\begin{abstract}
A positive correlation exists between the pathogenicity of bacteria and fungi when evaluated in the insect Galleria mellonella and mice. This work sought to determine whether fluctuations in the number of haemocytes and the proliferation of yeast cells in infected larvae could be used to determine the relative pathogenicity of a range of yeast isolates. Larvae were inoculated with $1 \times 10^{6}$ stationary-phase yeast cells and incubated in the dark at $30^{\circ} \mathrm{C}$ for $48 \mathrm{~h}$. The results indicated that larvae inoculated with the most pathogenic isolates (i.e. those capable of killing $>80 \%$ of infected larvae) showed a significant reduction in haemocyte density. Larvae inoculated with isolates of low pathogenicity (i.e. capable of killing $<20 \%$ of infected larvae) demonstrated only a small fluctuation in haemocyte numbers. The most pathogenic yeast isolates proliferated in the larvae, whereas the isolates of low pathogenicity did not. These results demonstrate a relationship between the ability of yeast isolates to kill larvae and changes in haemocyte density and yeast cell density in infected larvae. These end points may extend the applicability of the G. mellonella system for use with a wider range of microbial isolates.
\end{abstract}

(C) 2003 Éditions scientifiques et médicales Elsevier SAS. All rights reserved.

Keywords: Candida; Galleria; Haemocyte; Innate immunity; Pathogenicity; Virulence

\section{Introduction}

Conventional assays for evaluating the relative pathogenicity of microbial pathogens or mutants rely upon the use of a range of vertebrate species. Recent work has demonstrated that a strong correlation exists between the virulence of pathogens when determined in insects and mice [1-3]. The immune response of insects to pathogens shares a high degree of homology with the innate immune response of mammals [4-7], which plays a critical role in protecting the body from infection $[8,9]$. To date, much attention has focused on the use of larvae of Galleria mellonella (the greater wax moth) for evaluating the pathogenicity of bacteria [2], fungi $[1,10]$ and nematodes [11]. The use of insects for this purpose has many advantages over the conventional use of mammals, including reduced costs and faster results $[1,2]$.

The insect body cavity, or haemocoel, contains haemolymph, which serves a function analogous to blood in mammals in that it transports nutrients, waste products and

* Corresponding author. Tel.: +353-1-708-3859; fax: +353-1-708-3845.

E-mail address: kevin.kavanagh@may.ie (K. Kavanagh). signal molecules, although it plays no role in respiration. In addition, haemolymph contains cells (haemocytes) and antimicrobial peptides capable of immobilising and killing invading microorganisms $[12,13]$. The majority of haemocytes circulate freely within the haemolymph, but a significant number can be found associated with internal organs such as the fat body, trachea or digestive system [14]. At least six types of haemocytes have been identified in lepidopterous (e.g. G. mellonella) [15] and are classified as prohaemocytes, plasmatocytes, granulocytes (also known as granular cells), coagulocytes, spherulocytes and oenocytoids [16]. The plasmatocytes and granulocytes participate in phagocytosis, nodule formation and encapsulation [17], which are important elements of the insect's cellular defence against bacteria and unicellular fungi [18]. The insect immune response to microorganisms has been shown to involve a change in the circulating haemocyte population and synthesis of new haemolymph proteins [19].

The full potential of using insect larvae for evaluating microbial pathogenicity has yet to be exploited. At present, larval death is the end point chosen for use in in vivo pathogenicity assays $[1-3,10]$. Some microbes of low pathogen- 
Table 1

Yeast strains used in this study

\begin{tabular}{|c|c|c|}
\hline Yeast strains & Genotype/mutation & Reference \\
\hline C. albicans $\mathrm{MEN}$ & Clinical isolate & \\
\hline C. albicans mmu11 & Respiratory deficient $C$. albicans $\mathrm{MEN}$ & {$[20]$} \\
\hline C. albicans ATCC 44990 & $\mathrm{Ade}^{-}, \mathrm{thr}^{-}$ & {$[10]$} \\
\hline C. albicans ATCC 10231 & Clinical isolate & {$[10]$} \\
\hline C. albicans cybrid 3 & Mitochondrial transfer from 44990 to MMU11 & {$[20]$} \\
\hline C. albicans cybrid 26 & Mitochondrial transfer from 44990 to MMU11 & {$[20]$} \\
\hline C. albicans cybrid 27 & Mitochondrial transfer from 44990 to MMU11 & {$[20]$} \\
\hline C. albicans SC5314 & Clinical isolate & \\
\hline C. albicans JKC 19 & $\Delta c p h 1 / \Delta c p h 1$ & {$[21]$} \\
\hline C. albicans $\mathrm{CDH} 107$ & $\Delta$ caras $1 / \Delta$ caras 1 & {$[22]$} \\
\hline C. albicans CR216 & $\Delta c a c d c 35 / 4 c a c d c 35$ & {$[23]$} \\
\hline C. glabrata $\mathrm{NCPF} 4733$ & Clinical isolate & {$[10]$} \\
\hline C. parapsilosis NCPF3209 & Clinical isolate & {$[10]$} \\
\hline C. dubliniensis CD36 & Clinical isolate & {$[25]$} \\
\hline C. tropicalis NCPF3109 & Clinical isolate & {$[10]$} \\
\hline S. cerevisiae yjm 128 & Clinical isolate & {$[24]$} \\
\hline S. cerevisiae yjm $128 \rho$ & Respiratory deficient $S$. cerevisiae yjm 128 & \\
\hline
\end{tabular}

ATCC, American Type Culture Collection, Manasas, VA, USA; NCPF, National Collection of Pathogenic Fungi, Bristol, UK.

icity fail to kill larvae $[1,10]$ but may still evoke a low-level immune response, which may provide information on the mechanisms employed by the insect to eradicate infection and also allow a discrimination between pathogens of low pathogenicity. The work presented here sought to establish whether changes in the haemocyte density and fungal load in infected larvae could be used as indicators of fungal pathogenicity. These could have the potential to be used as end points for pathogenicity assays and provide information on the insect immune response to fungal pathogens.

\section{Materials and methods}

\subsection{Yeast strains and culture conditions}

The fungal strains used in this work are listed in Table 1. Cultures were grown to the stationary phase (approximately $1 \times 10^{8} / \mathrm{ml}$, typical viability $85-90 \%$ ) overnight in $50 \mathrm{ml}$ of YEPD (2\% (w/v) glucose (Sigma Aldrich Chemical Co., Dublin, Ireland), $2 \%(\mathrm{w} / \mathrm{v})$ bactopeptone (Oxoid Ltd., Basingstoke, England) and 1\% (w/v) yeast extract (Oxoid) in $100 \mathrm{ml}$ conical flasks at $200 \mathrm{rpm}$ in an orbital incubator at $30{ }^{\circ} \mathrm{C}$. Stocks of cultures were maintained on YEPD agar plates and subcultured every 4 weeks.

\subsection{Insect larvae}

Sixth instar larvae of G. mellonella (Lepidoptera: pyralidae, the greater wax moth) were obtained from the Mealworm Company (Sheffield, England) and stored in wood shavings in the dark at $15{ }^{\circ} \mathrm{C}$. Larvae weighing between 0.2 and $0.4 \mathrm{~g}$ were used in all experiments. Ten larvae were randomly chosen for each assay, and all experiments were performed on three independent occasions.

\subsection{Inoculation of $\mathrm{G}$. mellonella larvae}

The cell density of stationary-phase cultures was ascertained by haemocytometer count. Yeast cells were harvested by centrifugation at $2000 \times g$ in a Beckmann GC- 6 centrifuge, washed and diluted in phosphate-buffered saline (PBS, $\mathrm{pH} 7.2$ ) to a density of $5 \times 10^{7} / \mathrm{ml}$. Larvae were inoculated with $1 \times 10^{6}$ cells $/ 20 \mu \mathrm{l}$ through the last pro-leg into the haemocoel using a Myjector syringe (Terumo Europe, Leuven, Belgium) and incubated at $30{ }^{\circ} \mathrm{C}$ for $48 \mathrm{~h}$ in the dark [10]. Two sets of controls were employed in each assay. The first consisted of larvae that were not exposed to yeast (untouched control) while the second control consisted of larvae that were injected with $20 \mu \mathrm{l}$ sterile PBS (PBS injected). Larval death was determined by visual inspection (Fig. 1) and by the lack of movement when stimulated.

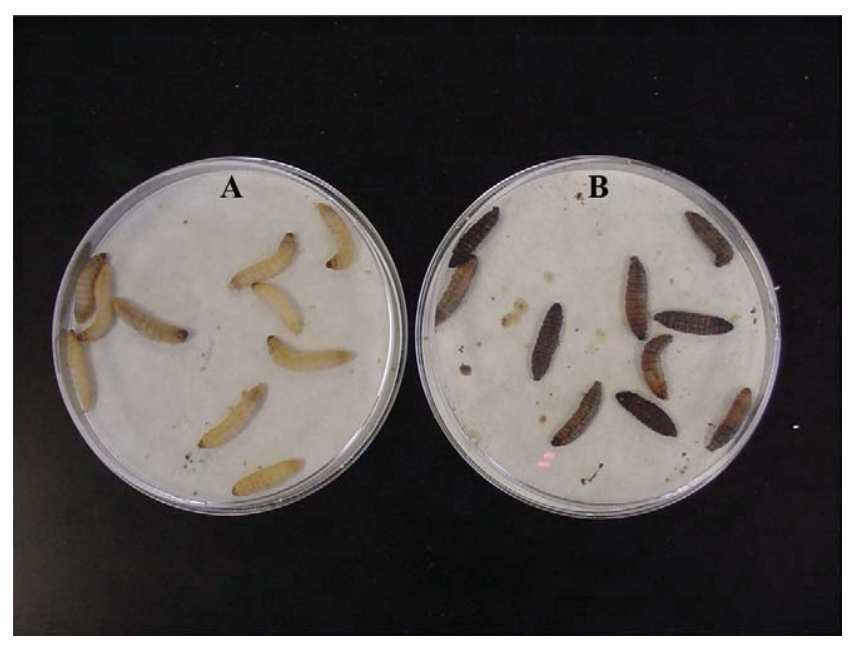

Fig. 1. Larvae of G. mellonella. (A) Control larvae, (B) larvae inoculated with $1 \times 10^{6}$ C. albicans MEN and incubated for $48 \mathrm{~h}$. Petri dish diameter is $8.5 \mathrm{~cm}$. 


\subsection{Determination of haemocyte density in $\mathrm{G}$. mellonella} larvae

Haemocyte density was assessed by bleeding three larvae per treatment into a pre-chilled test tube containing $4 \mathrm{mg}$ phenylthiourea (Sigma Aldrich) to prevent melanisation. Haemocytes were diluted in PBS containing $0.37 \%$ (v/v) mercaptoethanol (Sigma Aldrich), and their density was ascertained by haemocytometer count. No attempt to discriminate between the different haemocyte subtypes was made.

\subsection{Determination of fungal load in $\mathrm{G}$. mellonella}

Three inoculated larvae were homogenised in $3 \mathrm{ml}$ of sterile PBS. This was serially diluted with PBS, and $100-\mu 1$ aliquots of the resulting dilutions were plated on YEPD plates containing erythromycin $(1 \mathrm{mg} / \mathrm{ml})$ (Sigma Aldrich) to prevent bacterial overgrowth. These plates were incubated at $30{ }^{\circ} \mathrm{C}$ for $48 \mathrm{~h}$. The fungal load was calculated as the yeast cell density per larva and was based on the number of colonies that grew at specific dilutions.

\subsection{Statistical analysis}

All assays were performed on three independent occasions. Results presented in Fig. 2 are the mean percentage survival \pm standard deviation. Statistical analysis of data was performed using the Kruskal Wallis one-way ANOVA test and the Student-Newman Keuls method (Sigma Stat Statistical Analysis Package Version 1.00 (SPSS Inc., Chicago, IL, USA)). Values of $P<0.001$ are considered statistically significant.

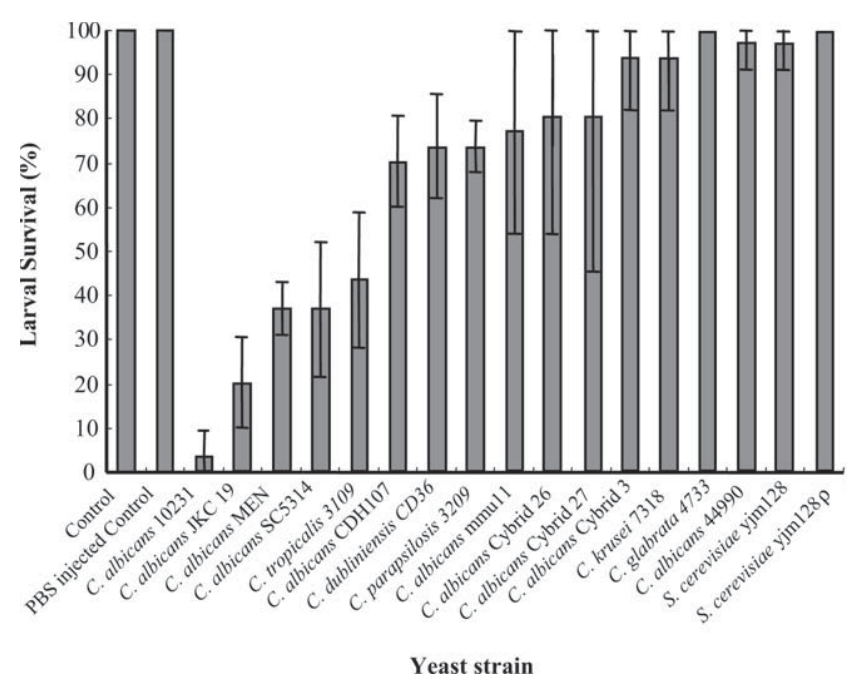

Fig. 2. Survival (\%) of G. mellonella larvae following inoculation with yeast isolates after $48 \mathrm{~h}$ incubation. All values are the mean \pm standard deviation of three independent determinations.

\section{Results}

\subsection{Determination of pathogenicity of yeast isolates in larvae of $\mathrm{G}$. mellonella}

Previous work has established the suitability of larvae of G. mellonella for detecting variations in the pathogenicity of yeast strains [10] and mutants of Candida albicans [1], and a positive correlation between the pathogenicity of mutants of C. albicans in insects and mice has been established [1]. The work presented here sought to determine the effect of a range of yeast isolates of differing pathogenicities on the haemocyte population and fungal loads of infected larvae. Consequently, the ability of isolates used in this work to kill G. mellonella larvae over a 48 -h period was assessed. Larvae were inoculated with yeast cells as described and incubated at $30{ }^{\circ} \mathrm{C}$ for $48 \mathrm{~h}$. The results (Fig. 2) demonstrate that the 17 yeast isolates employed here may be broadly differentiated into three groups, based on larval survival after $48 \mathrm{~h}$. The most pathogenic group gives a larval survival rate of less than $40 \%$ and contains C. albicans 10231, JKC 19, MEN and SC5314. The intermediate group contains four isolates, and these give a larval survival rate of between $40 \%$ and $80 \%$. This group contains the wild-type isolates Candida tropicalis, Candida dubliniensis and Candida parapsilosis and $C$. albicans CDH107. The third group contains the least pathogenic isolates, which give a larval survival rate of greater than $80 \%$. This group contains Candida krusei, Candida glabrata, C. albicans 44990 and cybrid 3, 26, 27 and MMU11. Saccharomyces cerevisiae yjm128 and its respiratorydeficient mutant are also in this group.

This result demonstrates that the isolates chosen for this work are of differing pathogenicity and that by using $G$. mellonella, they may be differentiated into three broad categories on the basis of their ability to kill larvae over a 48-h period.

3.2. An examination of haemocyte density in $\mathrm{G}$. mellonella larvae infected with yeast isolates of differing pathogenicity

Haemocytes represent the main element of the insect cellular response to infection, and fluctuations in haemocyte numbers have previously been shown to occur following infection with a range of microorganisms $[11,13]$. The objective of this work was to determine whether changes in haemocyte density in individual larvae could be correlated with larval survival following inoculation. The existence of such a correlation could give an indication of the insect immune response to yeast infection, as well as providing an additional end point for measuring the relative pathogenicity of fungal isolates.

Larvae were inoculated as described, and haemocytes were removed and quantified after $48 \mathrm{~h}$ incubation. Control larvae had a mean haemocyte density of $9.67 \times 10^{6} /$ larva after $48 \mathrm{~h}$ incubation, whereas those infected with isolates of high pathogenicity (e.g. C. albicans 10231 or MEN) showed 
Table 2

Fluctuations in haemocyte density in larvae inoculated with yeast isolates

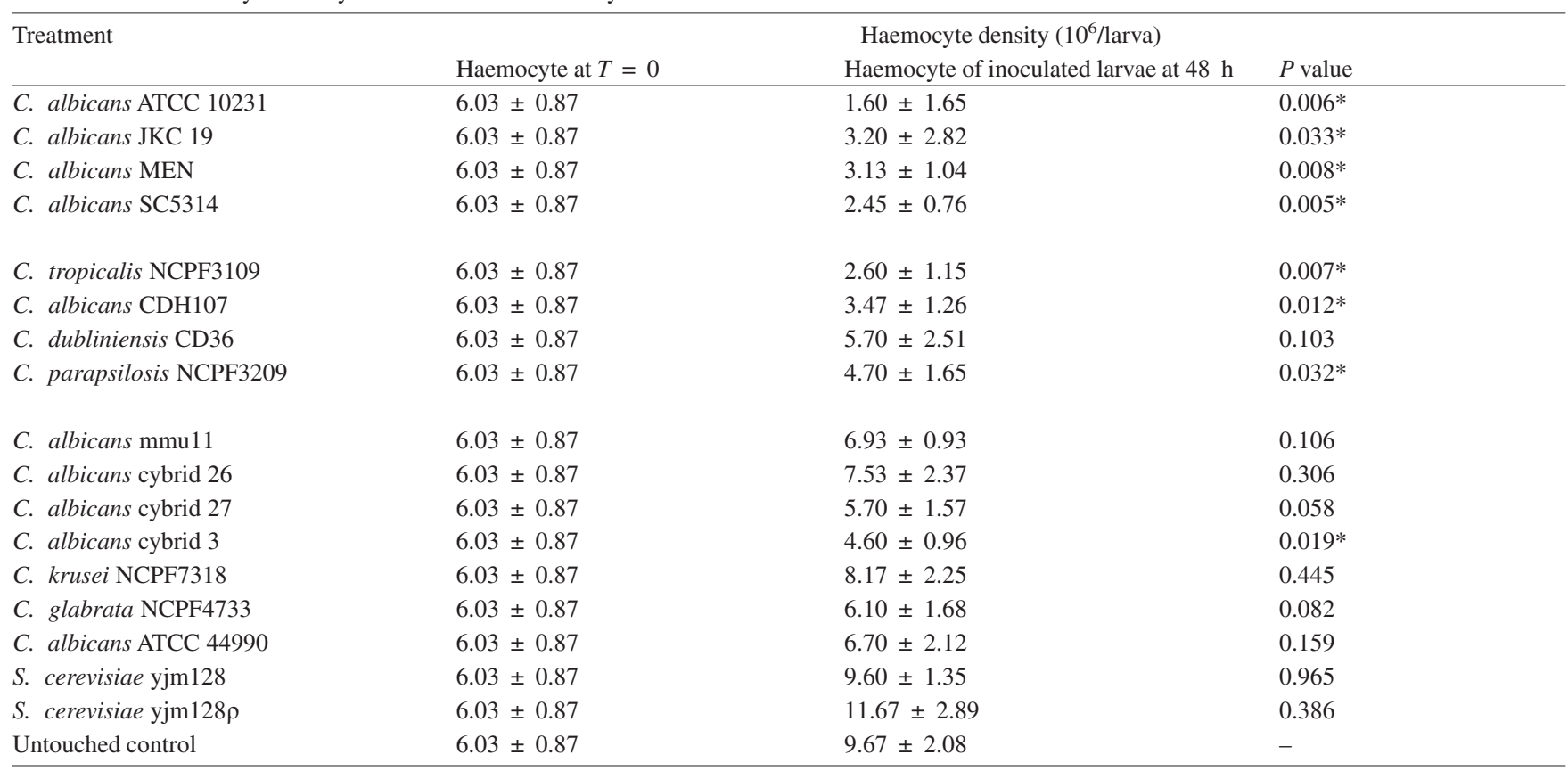

* Statistically significant using $t$-test. Values are compared with the haemocyte density in untouched larvae at $48 \mathrm{~h}$.

a haemocyte density of approximately 1.6-3.2 $\times 10^{6} /$ larva (Table 2). Larvae inoculated with isolates of low pathogenicity (e.g. C. glabrata or C. albicans 44990) showed a smaller decrease in haemocyte density than that observed in larvae inoculated with isolates of high pathogenicity, while those inoculated with $S$. cerevisiae 128 demonstrate an increase in haemocyte numbers (Table 2).

A linear relationship appears to exist between the haemocyte density in individual larva and the survival rate of the larvae. Larvae inoculated with isolates of high pathogenicity have a reduced haemocyte density, while those inoculated with an isolate of low pathogenicity display only a small variation in haemocyte density compared to uninfected controls (Fig. 3).

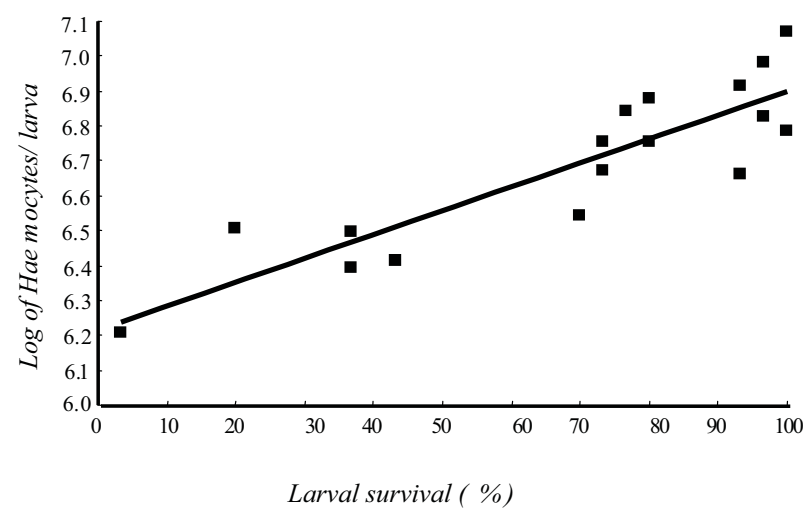

Fig. 3. Haemocyte density in G. mellonella larvae inoculated with yeast isolates of differing pathogenicity $\left(y=0.0069 x+6.2136, R^{2}=0.7857\right)$.

\subsection{Fungal load in infected $\mathrm{G}$. mellonella larvae}

The insect immune system is very effective in dealing with invading pathogens [26], and for a pathogen to survive and proliferate, it must overcome a rapid innate immune response [8]. Experiments were performed to determine whether fungal cells of pathogenic isolates proliferated in the insect haemolymph and whether this proliferation could be correlated with the ability to kill. Larvae were inoculated at a density of $1 \times 10^{6}$ cells/larva as described, and the resulting number of yeast cells per larva (termed the 'fungal load') after $48 \mathrm{~h}$ incubation was evaluated by homogenising larvae and plating out on medium supplemented with erythromycin. The results (Table 3) demonstrate that the most pathogenic isolates proliferate upon inoculation and give the highest yeast cell density per larva $\left(3-8 \times 10^{7}\right.$ cells $)$, while isolates of low pathogenicity (e.g. C. albicans cybrids 3,26 and 27) fail to proliferate significantly and, after $48 \mathrm{~h}$ incubation, have a density substantially lower than the initial dose. The only exceptions to this are $C$. albicans MMU11 and ATCC 44990 and C. krusei NCPF 7318, which show a small degree of proliferation (Table 3).

This result indicates that a linear relationship exists between the ability of yeast isolates to kill larvae and the resulting numbers of fungal cells per larva (Fig. 4). The most pathogenic isolates proliferate to the greatest extent in the larvae. This may indicate that they can avoid or tolerate the immune response, which may retard the proliferation of isolates of low pathogenicity. 
Table 3

Variations in fungal load in larvae inoculated with yeast isolates

\begin{tabular}{|c|c|c|c|}
\hline \multirow[t]{2}{*}{ Treatment } & \multicolumn{3}{|c|}{ Fungal load $\left(10^{6} /\right.$ larva $)$} \\
\hline & Initial inoculum & $48 \mathrm{~h}\left(\times 10^{6}\right)$ & $P$ value \\
\hline C. albicans ATCC 10231 & $1 \times 10^{6}$ & $80.82 \pm 13.64$ & $<0.001 *$ \\
\hline C. albicans JKC 19 & $1 \times 10^{6}$ & $34.15 \pm 17.52$ & $0.031 *$ \\
\hline C. albicans $\mathrm{MEN}$ & $1 \times 10^{6}$ & $35.50 \pm 13.60$ & $0.012 *$ \\
\hline C. albicans SC5314 & $1 \times 10^{6}$ & $50.52 \pm 20.40$ & $0.014 *$ \\
\hline C. tropicalis NCPF3109 & $1 \times 10^{6}$ & $66.80 \pm 4.60$ & $<0.001 *$ \\
\hline C. albicans $\mathrm{CDH} 107$ & $1 \times 10^{6}$ & $5.30 \pm 4.81$ & 0.196 \\
\hline C. dubliniensis CD36 & $1 \times 10^{6}$ & $36.47 \pm 11.54$ & $0.006^{*}$ \\
\hline C. parapsilosis NCPF3209 & $1 \times 10^{6}$ & $36.57 \pm 3.86$ & $<0.001 *$ \\
\hline C. albicans mmu11 & $1 \times 10^{6}$ & $3.01 \pm 3.33$ & 0.356 \\
\hline C. albicans cybrid 26 & $1 \times 10^{6}$ & $0.05 \pm 0.00$ & $<0.001 *$ \\
\hline C. albicans cybrid 27 & $1 \times 10^{6}$ & $0.05 \pm 0.05$ & $<0.001 *$ \\
\hline C. krusei NCPF7318 & $1 \times 10^{6}$ & $1.32 \pm 0.03$ & $<0.001 *$ \\
\hline C. glabrata $\mathrm{NCPF} 4733$ & $1 \times 10^{6}$ & $0.10 \pm 0.05$ & $<0.001 *$ \\
\hline C. albicans ATCC 44990 & $1 \times 10^{6}$ & $5.65 \pm 9.53$ & 0.445 \\
\hline S. cerevisiae yjm 128 & $1 \times 10^{6}$ & $0.22 \pm 0.06$ & $<0.001 *$ \\
\hline S. cerevisiae yjm 128 petite & $1 \times 10^{6}$ & $0.12 \pm 0.06$ & $<0.001 *$ \\
\hline
\end{tabular}

${ }^{*}$ Statistically significant using $t$-test. Values are compared with initial fungal load $\left(1 \times 10^{6}\right)$.

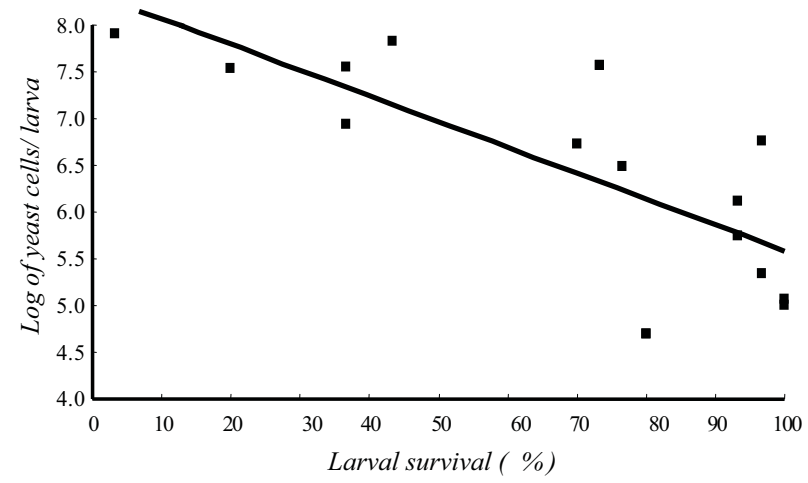

Fig. 4. Yeast cell density in G. mellonella larvae inoculated with yeast isolates of differing pathogenicity $\left(y=-0.0276 x+8.3485, R^{2}=0.5188\right)$.

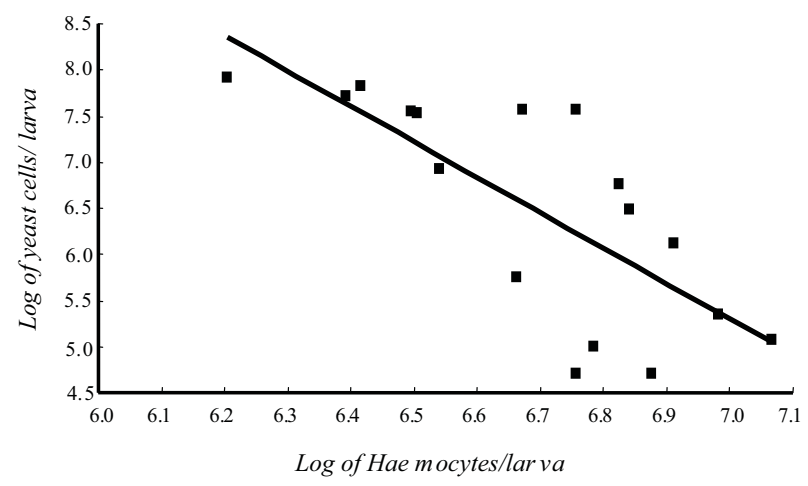

Fig. 5. Relationship between yeast cell density and haemocyte density in inoculated $G$. mellonella larvae $\left(y=-3.839 x+32.172, R^{2}=0.5619\right)$.
An examination of the haemocyte density and fungal loads of larvae inoculated with yeast isolates of different pathogenicities demonstrates that a high haemocyte density is associated with a low fungal load (Fig. 5), and a high fungal load is found in larvae with a relatively low haemocyte density.

\section{Discussion}

Larvae of G. mellonella have been used to evaluate the relative pathogenicity of yeast species [10], and a correlation between the pathogenicity of C. albicans mutants in Galleria and mice has been established [1]. The ability of G. mellonella larvae to detect differences in the pathogenicity of lipopolysaccharide-deficient mutants of Pseudomonas aeruginosa has also been demonstrated [27], and a strong correlation between the virulence of $P$. aeruginosa in Galleria larvae and in mice has been demonstrated [2]. Larvae of G. mellonella have been used to assess the virulence of Bacillus thuringiensis and Bacillus cereus, and a good agreement between the results obtained in insects and mice has been established [3]. The survival of larvae following inoculation with microbes has been used as the sole end point for in vivo pathogenicity assays. In the work presented here we sought to establish whether parameters such as changes in haemocyte density or fungal load could be used as indicators of fungal pathogenicity. Monitoring of these parameters could also offer an insight into the immune response of $G$. mellonella larvae to fungal pathogens. 
In vivo pathogenicity assays using $G$. mellonella larvae demonstrated that the yeast isolates used in this study could be differentiated into three broad categories based on their ability to kill larvae after $48 \mathrm{~h}$ incubation. The most pathogenic isolates produced a survival rate of less than $40 \%$ after $48 \mathrm{~h}$ incubation, whereas the least pathogenic isolates gave a larval survival rate of in excess of $80 \%$ over the same time frame.

Fluctuations in the haemocyte density of larvae were examined, since haemocyte numbers have been shown to vary in insects infected with microorganisms [11,13] Examination of changes in haemocyte density in larvae inoculated with the different isolates used in this study indicate that a low survival rate is correlated with a reduced haemocyte density, whereas a high survival rate is correlated with a haemocyte density close to that of control larvae. Changes in haemocyte density following microbial challenge have been observed previously. In the case of G. mellonella infected with Stinerema nematodes, there is a decrease in haemocyte numbers in the first $4 \mathrm{~h}$ followed by a gradual increase over the next $12 \mathrm{~h}$ to $160 \%$ of the control. Subsequently, numbers decline to less than $5 \%$ of the control haemocyte density, which is associated with the death of the larvae [11]. The initial decline in haemocyte numbers has been attributed to the formation of clumps consisting of haemocytes and invading microbes [28]. The subsequent rise in their numbers is thought to be due to the release of haemocytes bound to internal organs.

Analysis of the fungal load of larvae inoculated with $1 \times$ $10^{6}$ yeast cells indicates a high survival rate in cases where there is little or no yeast proliferation, while a low survival rate is associated with extensive yeast proliferation. Haemocytes represent one element of the insect immune response to infection and counter the entry of microbial pathogens by phagocytosis or encapsulation [26]. It is possible that those strains that proliferate to the greatest extent in the larvae are capable of evading neutralisation by the haemocyte population and subsequently replicate. In addition to cells of the immune system, the insect haemolymph also contains trehalose, which may represent a nutrient source for the growth of yeast cells that survive the initial immune response [29].

Da Silva et al. [30] noted that a reduction in the number of C. albicans in the haemolymph of Culex quinquefasciatus (mosquito) and a concomitant rise in the haemocyte density was indicative of an effective cellular immune response. In the work presented here, an effective immune response is apparent following inoculation of larvae with yeast isolates of low pathogenicity, whereas isolates of high pathogenicity overcome the cellular response, leading to a decline in haemocyte numbers and an increase in yeast cells in the haemolymph.

A correlation exists between larval survival and changes in haemocyte density and fungal load (Figs. 3-5). Yeast isolates of high pathogenicity can be identified by a low haemocyte density (1.6-3.2 × 10\%/larva) and a high fungal load $\left(3-8 \times 10^{7} /\right.$ larva) in infected larvae. Isolates of low pathogenicity are identifiable by a high haemocyte density (up to $1 \times 10^{7} /$ larva) and fungal load similar to or less than the original inoculum dose. This demonstrates that yeast isolates capable of killing larvae proliferate in the haemolymph and lead to a reduction in the number of haemocytes. Given the correlation between changes in haemocyte density and fungal load and pathogenicity, it may be possible to use these parameters as end points in assays using G. mellonella to evaluate fungal pathogenicity.

The insect immune system displays a high degree of structural and functional homology with the innate immune response of mammals $[8,9,31]$. Consequently it has proven possible to establish a correlation between the pathogenicity of bacteria and fungi in $G$. mellonella larvae and mice [1,2]. The use of insects in place of mammals for routine testing of microbial pathogens (or their mutants) would reduce cost, labour and the need to use the same number of mammals as used at present. It has been demonstrated previously that larval death can be employed as an end point for this type of assay $[1-3,10,11,13]$. The work presented here demonstrates two further end points that may extend the applicability of the G. mellonella model system.

\section{Acknowledgements}

The authors are grateful to Dr. Malcolm Whiteway for the gift of isolates C. albicans SC5314, JKC 19, CDH107 and CR216, to Dr. Derek Sullivan for the gift of C. dubliniensis CD36 and to Dr. Karl Clemons for the gift of S. cerevisiae yjm128. This work was supported by funding from the HEA under PRTLI 3.

\section{References}

[1] M. Brennan, D.Y. Thomas, M. Whiteway, K. Kavanagh, Correlation between virulence of Candida albicans mutants in mice and Galleria mellonella larvae, FEMS Immunol. Med. Microbiol 34 (2002) 153157.

[2] G. Jander, L.G. Rahme, F.M. Ausubel, Positive correlation between virulence of Pseudomonas aeruginosa mutants in mice and insects, J. Bacteriol 182 (2000) 3843-3845.

[3] S. Salamitou, F. Ramisse, M. Brehelin, D. Bourguet, N. Gilois, M. Gominet, E. Hernandez, D. Lereclus, The plcR regulon is involved in the opportunistic properties of Bacillus thuringiensis and Bacillus cereus in mice and insects, Microbiology 146 (2000) 2825-2832.

[4] D. Wittwer, A. Franchini, E. Ottaviani, A. Wiesner, Presence of IL-1 and TNF like molecules in Galleria mellonella (Lepidoptera) haemocytes and in an insect cell line from Estimene acraea (Lepidoptera), Cytokine 11 (1999) 637-642.

[5] O. Takeuchi, A. Shizuo, Toll-like receptors; their physiological role and signal transduction system, Int. Immunopharmacol 1 (2001) 625635.

[6] F.L. Rock, G. Hardiman, J.C. Timans, R.A. Kastelein, J.F. Bazan, A family of human receptors structurally related to Drosophila, Dev. Biol 95 (1998) 588-593. 
[7] L. Zhao, M.R. Kanost, In search of a function for Hemolin, a hemolymph protein from the immunoglobulin superfamily, J. Insect Physiol 42 (1996) 73-77.

[8] P. Vilmos, E. Kurucz, Insect immunity: evolutionary roots of the mammalian innate immune system, Immunol. Lett 62 (1998) 59-66.

[9] M. Salzet, Vertebrate innate immunity resembles a mosaic of invertebrate immune responses, Trends Immunol 22 (2001) 285-288.

[10] G. Cotter, S. Doyle, K. Kavanagh, Development of an insect model for the in vivo pathogenicity testing of yeasts, FEMS Immunol. Med. Microbiol 27 (1999) 163-169.

[11] V. Matha, Z. Mraeck, Changes in haemocyte counts in Galleria mellonella (Lepidoptera: Galleriidae) larvae infected with Steinernema Sp. (Nematoda: Steinernematidae), Nematologica 30 (1984) 86-89.

[12] M.D. Lavine, M.R. Strand, Insect hemocytes and there role in immunity, Insect Biochem. Mol. Biol. 32 (2002) 1295-1309.

[13] D. Morton, G. Dunphy, J. Chadwick, Reactions of haemocytes of immune and non-immune Galleria mellonella larvae to Proteus mirabilis, Dev. Comp. Immunol 11 (1987) 47-55.

[14] N. Ratcliffe, Invertebrate immunity - a primer for the non-specialist, Immunol. Lett 10 (1985) 253-270.

[15] H.G. Boman, D. Hultmark, Cell-free immunity in insects, Annu. Rev. Microbiol. 41 (1987) 103-126.

[16] C.D. Price, N.A. Ratcliffe, A reappraisal of insect haemocyte classification by the examination of blood from fifteen insect orders, Z . Zellforsch. Mikrosk. Anat. 147 (1974) 537-549.

[17] S. Tojo, F. Naganuma, K. Arakawa, S. Yokoo, Involvement of both granular cells and plasmatocytes in phagocytic reactions in the greater wax moth, Galleria mellonella, J. Insect Physiol. 46 (2000) 11291135 .

[18] J.B. Walters, N.A. Ratcliffe, Studies on the in vivo cellular reactions of insects: fate of pathogenic and non-pathogenic bacteria in Galleria mellonella nodules, J. Insect Physiol. 29 (1983) 417-424.

[19] P. Engstrom, A. Carlsson, A. Engstrom, Z. Tao, H. Bennich, The anti-bacterial effect of attacins from the silk moth Hyalophora cecropia is detected against the outer membrane of Escherichia coli, EMBO J. 3 (1984) 3347-3351.
[20] P. Geraghty, K. Kavanagh, Disruption of mitochondrial function in Candida albicans leads to reduced cellular ergosterol levels and elevated growth in the presence of amphotericin B, Arch. Microbiol. 179 (2003) 295-300.

[21] H.J. Lo, J.R. Kohler, B. DiDomenico, D. Loebenberg, A. Cacciapuoti, G.R. Fink, Nonfilamentous C. albicans mutants are avirulent, Cell 90 (1997) 939-949.

[22] E. Leberer, D. Harcus, D. Dignard, S. Ushinsky, D.Y. Thomas, K. Schroppel, Signaling through Ras links cellular morphogenesis to virulence in the pathogenic fungus Candida albicans, Mol. Microbiol. 42 (2001) 673-687.

[23] C.R.C. Rocha, K. Schroppel, D. Harcus, A. Marcil, D. Dignard, B.N. Taylor, D.Y. Thomas, M. Whiteway, E. Leberer, Signalling through adenylyl cyclase is essential for hyphal growth and virulence in the pathogenic fungus Candida albicans, Mol. Biol. Cell 12 (2001) 3631-3643.

[24] J.K. Byron, K.V. Clemons, J.H. McCusker, R.W. Davis, D.A. Stevens, Pathogenicity of Saccharomyces cerevisiae in complement factor five deficient mice, Infect. Immun 63 (1995) 478-485.

[25] G.D. Gilfillan, D.J. Sullivan, K. Haynes, Candida dubliniensis: Phylogeny and putative virulence factors, Microbiology 144 (1998) 829838.

[26] J. Hoffman, Innate immunity of insects, Curr. Opin. Immunol 7 (1995) 4-10.

[27] G. Dunphy, D. Morton, A. Kropinski, J. Chadwick, Pathogenicity of lipopolysaccharide mutants of Pseudomonas aeruginosa for larvae of Galleria mellonella: bacterial properties associated with virulence, J. Invert. Pathol. 47 (1986) 48-55.

[28] S.J. Gagen, N.A. Ratcliffe, Studies on the in vivo cellular reactions and fate of injected bacteria in Galleria mellonella and Pieris brassicae larvae, J. Invert. Pathol 28 (1976) 17-24.

[29] J.L. Nation, Insect Physiology and Biochemistry, CRC Press, Washington, DC, 2002, pp. 301-326.

[30] J.B. Da Silva, M.R. De Albuquerque, E. De Araujo, C.A. Peixoto, H. Hurd, Immune defence mechanisms of Culex quinquefasciatus (Diptera: Culicidae) against Candida albicans infection, J. Invert. Pathol. 76 (2000) 257-262.

[31] J. Klein, Homology between immune responses in vertebrates and invertebrates: does it exist? Scand. J. Immunol 46 (1997) 558-564. 